\title{
Possibilidades de contribuição do farmacêutico para a promoção da saúde
}

\author{
How pharmacists can contribute to health promotion
}

Fabiola Sulpino Vieira ${ }^{1}$

\footnotetext{
'M inistério da Saúde, Secretaria de Ciência, Tecnologia elnsumos Estratégicos, Departamento deAssistência Farmacêutica. Esplanada dos M inistérios Bloco G Ed Sede 8 andar sala 829. 70058-900 Brasília DF

fabiolasulpino@uol.com.br
}

Abstract M edicinesareconsidered themain therapeutical tool for the treatment or maintenance of public health conditions. However, the symbolism in wich they are cloaked and thus their use by society, has contributed to the emergence of many adverse events with high impact on health and health systems costs. Thus, rational drug use promotion is an important tool to eliminate or minimize the problem. This is where the pharmacists can play an important role because this is a pertinent subject in their field of performance. Their participation in multidisciplinary teamsadds value to health services and contributes to heal th promotion. This articleaddresses theseissues and how pharmacists can contribute to health promotion. Key words Rational druguse, Pharmacist, Health promotion, Pharmaceutical care
Resumo Os medicamentos são considerados a principal ferramenta terapêutica para recuperação ou manutenção das condições de saúde da população. No entanto, o si mbolismo de que eles são revestidos e, conseqüentemente, o uso dos mesmos pela sociedade, tem contribuído para o surgimento demuitoseventos adversos, com elevado impacto sobre a saúde e custos dos sistemas. Assim, a promoção do uso racional dos medicamentoséuma ferramenta importante de atuação junto à sociedade, para senão eliminar, minimizar o problema. N este sentido, o farmacêutico pode contribuir sobremaneira, já que este é assunto pertinente a seu campo de atuação. Sua participação em equipes multidisplinares acrescenta valor aos serviços e contribui para a promoção da saúde. Este artigo trata destasquestõese discuteas possi bilidades desta contribuição.

Palavras-chaves U so racional de medicamentos, Farmacêutico, Promoção da saúde, Atenção farmacêutica 
Introdução

A prevenção e o tratamento de doenças exigem infra- estrutura adequada, assi m como educação apropriada. A pós estas medidas, os medicamentos eas vacinas têm o potencial de conferir grandes benefícios à população ${ }^{1}$. No entanto, 0 simbolismo de que se revestem os medicamentos na sociedade tem contribuído para a utilização irracional dos mesmos.

Segundo a Sociedade Brasileira de Vigilância de M edicamentos (Sobravime)2: o medicamento como parte do complexo médico-industrial influi na percepção da saúde e da doença, tanto nos profissionais desaúde quanto na sociedade. 0 medicamento não se apresenta sozinho - como sustância química - mas está acompanhado por um cotejo de publicidade, informação, brindes, estudos etc., que vai configurando uma forma de pensar.

M ais adiante, a Sobravime complementa: a medicalização de um número crescente de problemas da vida não constitui apenas um processo individual em que o futuro consumidor de cuidados médicos se convence de que se encontra na anormalidade. Trata-se também de um fenômeno cole tivo próprio das sociedades em crescimento que criam uma raridade do normal, afirmando que esteé o melhor.

A medicalização de aspectos da vida chega a ter dimensão tal, que hoje um novo conceito envolvendo medicamentos começa a ser utilizado: "fármacos do estilo de vida" (em inglês, "lifestyle drugs"). Este termo, conforme definição de Flower $^{3}$, diz respeito àqueles usados para satisfazer a objetivo não médico ou não relacionado à saúde.

É preocupante esta forma de aceitação dos medicamentos pela sociedade, tendo em vista o volume de produtos farmacêuticos comercializados. Dados da Associação Brasileira de Redes de Farmácias e D rogarias ${ }^{4}$ referem que, em 2003, estes estabelecimentos venderam aproximadamente 3,6 bilhões de reais em medicamentos (venda nominal), contabilizando o total de $260 \mathrm{mil}$ clientes atendidose 669 milhões deunidades vendidas. Se houvesse dados de todos os medicamentos dispensados pelo Sistema Ú nico de Saúde (SUS), ter-se ia como estimar o número de unidades farmacêuticas totais consumidas no país. Só para se ter uma idéia, se considerarmos que somente o M inistério da Saúde ${ }^{5}$, em 2002, despendeu 1,8 bilhões de reais para a aquisição de medicamentos para uso em unidades ambulatoriais ou repasse de recursos para a compra descentralizada destes, podemos observar que este número de unidades farmacêuticas consu- midas aumenta significativamente com a soma da dispensação pública (o valor referido não considera o recurso gasto em medicamentos empregados em ambiente hospitalar). Portanto, estes são valores que chamam a atenção para o volume de medicamentos que está sendo utilizado pela população e preocupam no sentido de que pode haver muita irracionalidade no seu uso, aumentando o risco de surgimento de morbidades relacionadas aos medicamentos e até mesmo da ocorrência de mortes, com elevação dos custos para os sistemas de saúde ${ }^{6}$.

Estima-se que nos EUA as reações adversas a medicamentos sejam a quarta ou a sexta causa demorte em hospitais, excedendo as mortes causadas por pneumonia e diabetes ${ }^{7}$. A morbimortalidade por esta causa é considerada comum eo custo estimado é da ordem de 136 bilhões de dólares ao ano ${ }^{8}$.

Uma revisão sistemática sobre os aten dimentos de emergência relacionados ao uso de medicamentos $s^{9}$ considerou dados de oito ensaios retrospectivos e quatro prospectivos. Os resultados indicaram que $28 \%$ de todos os atendimentos de emergência estão relacionados aos medicamentos. Destes atendimentos, $70 \%$ diziam respeito a situações evitáveis e $24 \%$ deles resultaram em internação hospitalar. Esta mesma pesquisa revela que os problemas mais comuns relacionados aos medicamentos são: as reações adversas, a não aderência ao tratamento e a prescrição inadequada.

Outro estudo ${ }^{10}$ mostra que os eventos adversos relacionados a medicamentos aumentam em 1,88 o risco de mortalidade e que $27 \%$ dos eventos relatados são atribuídos à negligência. Segundo o autor, uma solução viável para o problema é aumentar a colaboração entre médico e farmacêutico.

Desta forma, considerando a importância do tema e a escassez de informações sobre o uso de medicamentos no país, este artigo faz considerações sobre as possibilidades de contribuição do farmacêutico para a melhoria da utilização de medicamentos pela sociedade.

\section{O papel do farmacêutico ao longo do século XX}

Os autores Hepler \& Strand ${ }^{11}$ realizaram uma análise sobre os três períodos que consideram mais importantes da atividade farmacêutica no século XX, definindo-os como: o tradicional, o de transição e o de desenvolvimento da atenção 
ao paciente. 0 papel tradicional foi desenvolvido pelo boticário que preparava e vendia os medicamentos, fornecendo orientações aos seus clientes sobre o uso dos mesmos. Era comum prescrevê-los.

Conformea indústria farmacêutica começou a se desenvolver, este papel do farmacêutico paulatinamente foi diminuindo. Começa assim o período de transição. As atividades farmacêuticas voltaram-se principalmente para a produção demedicamentos numa abordagem técnicoindustrial. Os países do Primeiro Mundo concentraram-se no desenvolvimento de novos fármacos e o Brasil, que possui um parque industrial farmacêutico predominantemente multinacional, trabalhou a tecnologia farmacêutica adaptando as fórmulas às condições climáticas do país.

A publicação da Lei 5.991/73'2, queainda está em vigor, conferiu às atividades farmacêuticas um enfoque mercantilista. Qualquer empreendedor pode ser proprietário de uma farmácia ou drogaria, desde que conte com um profissional farmacêutico que se responsabilize tecnicamente pelo estabelecimento ${ }^{13}$. Este é o marco da perda do papel social desenvolvido pela farmácia. 0 estabelecimento comercial farmacêutico voltou-se para o lucro e o farmacêutico começou a perder autonomia para o desempenho de suas atividades. 0 profissional passou a atuar como mero empregado da farmácia ou drogaria, perdeu o respeito da sociedade erefugiou-se em outras atividades, distanciando-se de seu papel de agente de saúde. Com isto, ampliaram-se os espaços para a obtenção de lucros desenfreados através da "empurroterapia" ${ }^{14}$ e da propaganda desmedidas ${ }^{15}$.

O medicamento passou a ser visto com uma solução "mágica" para todos os problemas humanos, assumindo o conceito de bem de consumo em detrimento ao de bem social.

Mas, enfim, o farmacêutico em meio a uma grave crise de identidade profissional iniciou sua reação fazendo nascer nos anos 60 a prática da farmácia clínica ${ }^{16}$. Passou a se conscientizar do seu papel para a saúde pública. A prática farmacêutica orienta-se para a atenção ao paciente e o medicamento passa a ser visto como um meio ou instrumento para se alcançar um resultado, seja este paliativo, curativo ou preventivo. Ou seja, a finalidade do trabal ho deixa de focalizar o medicamento enquanto produto farmacêutico e passa a ser direcionada ao paciente, com a preocupação de que os riscos inerentes à utilização deste produto sejam minimizados.

O lamentável desastreocorrido em 1962, em virtude do uso da talidomida por gestantes, ocasionando uma epidemia de focomelia, desencadeou um novo olhar sobre o uso dos medicamentosefoi o marco para o surgimento das ações de farmacovigilância ${ }^{17}$. Passou-se então ao período de desenvolvimento da atenção ao paciente.

Os países começaram a se preocupar com a promoção do uso racional dos medicamentos ${ }^{18}$, motivados pela publicação de documentos pela Organização Mundial da Saúde (OMS). 0 primeiro passo neste sentido foi a introdução do conceito de medicamentos essenciais em $1977^{19}$.

No Brasil, a partir de meados dos anos 1990, a presença marcante das ações dos conselhos de farmácia evigilância sanitária em estabelecimentos comerciais farmacêuticos está mudando o panorama nacional. A exigência da presença do farmacêutico no setor privado está trazendo-o para o desempenho de suas funções em farmácias e drogarias.

O mesmo está ocorrendo no serviço público, embora de forma muito mais lenta. Hoje, mais do que nunca, é possível encontrar farmacêuticos desempenhando funções dentro das secretarias municipais da saúde, mas o número de profissionais está muito aquém das reais necessidades. Ainda não está garantida a sua presença em todas as unidades básicas de saúde, mesmo existindo dispositivo legal que determine isto ${ }^{12}$. No entanto, temos que refletir que mudanças estão ocorrendo e que acenam para a melhora dos serviços oferecidos à população.

No sentido da necessidade defomento à qualidade da assistência farmacêutica, em 1990, $\mathrm{He}$ pler \& Strand ${ }^{11}$ expuseram sua preocupação com os problemas que os medicamentos podem causar em relação à diminuição da qualidade de vida do paciente. Identificaram oito categorias de problemas relacionados aos medicamentos. Este foi o impulso inicial para o surgimento de uma nova prática, a atenção farmacêutica.

0 conceito proposto de atenção farmacêutica pelos autores citados difundiu-se mundialmente: "é a provisão responsável do tratamento farmacológico com o propósito de alcançar resultados concretos que melhorem a qualidade de vida do paciente".

Em 1993, a Organização Mundial da Saúde ${ }^{20}$ publicou o documento conhecido como "Declaração de Tóquio", resultado do trabal ho do grupo que se reuniu nesta cidade para discutir o papel do farmacêutico no sistema de aten ção à saúde. Abre-se espaço sem precedentes para a ampliação da prática farmacêutica.

Este novo modo de pensar a aten ção ao paci- 
ente pelo farmacêutico tem se difundido com rapidez e tem sido debatido com relação às potencialidades para a saúde pública.

O farmacêutico está voltando a cumprir o seu papel perante a sociedade, corresponsabilizando-sepelo bem estar do pacienteetrabal hando para que este não tenha sua qualidade de vida comprometida por um problema evitável, decorrentedeuma terapia farmacológica. Esteéum compromisso de extrema relevância, já que os eventos adversos a medicamentos são considerados hoje uma patologia emergente ${ }^{21}$ e são responsáveis por grandes perdas, sejam estas de ordem financeira ou de vida.

No Brasil, a Organização Pan-Americana da Saúde ${ }^{22}$ (OPAS) comandou uma oficina de trabalho para discutir a atenção farmacêutica. 0 grupo constituído para este fim produziu o documento "Proposta de Consenso Brasileiro de Atenção Farmacêutica", que tem por objetivo uniformizar os conceitos e a prática profissional no país.

A proposta de consenso defende que a prática da atenção farmacêutica deveestar orientada para a educação em saúde, orientação farmacêutica, dispensação, atendimento e acompanhamento farmacêutico, registro sistemático das atividades, mensuração e avaliação dos resultados. 0 propósito da atenção farmacêutica é reduzir a morbimortalidade relacionada aos medicamentos.

\section{Contribuição da profissão farmacêutica para a promoção da saúde}

Dentro deste novo contexto da prática farmacêutica, no qual a preocupação com o bem estar do paciente passa a ser a viga mestra das ações, 0 farmacêutico assume papel fundamental, somando seus esforços aos dos outros profissionais de saúde e aos da comunidade para a promoção da saúde.

Os autores James \& Rovers ${ }^{23}$ identificaram quatro categorias de iniciativas que podem ser implantadas pelos farmacêuticos para a melhoria do estado de saúde da comunidade:

- Acompanhamento e educação do e para o paciente;

- Avaliação dos seus fatores de risco;

- Prevenção da saúde;

- Promoção da saúdee vigilância das doenças.

Ainda segundo os autores acima, a promoção da saúde pode ser feita através de três domínios que dão suporte aos serviços oferecidos à população:
- Disposição deserviços de prevenção clínica;

- Vigilância e publicações em saúde pública e

- Promoção do uso racional de medicamentos pela sociedade.

A abordagem de James \& Rovers ${ }^{23}$ está alinhada aos tópicos relacionados pela OM S ${ }^{24} \mathrm{em}$ relação a medidas que podem ser adotadas para a promoção da saúde.

Transpondo para a nossa realidade as estratégias defendidas mundial mente, é possível dizer que o farmacêutico (da drogaria, farmácia comercial ou farmácia privativa dos hospitais e unidades ambulatoriais de saúde) pode trabaIhar sob três pontos básicos: reorientando o serviço de farmácia, desenvolvendo as habilidades da comunidade e incentivando os indivíduos à ação comunitária.

\section{Reorientação do serviço de farmácia}

Estudo realizado em farmácias e drogarias sobre o manejo da diarréia utilizando a terapia de re-hidratação oral concluiu que estes estabelecimentos podem se tornar postos avançados desaúdequando bem utilizados ${ }^{25}$. Estepotencial acena para 0 desempenho de um importante papel para a saúde pública, diferente da prática rotineira até então observada.

Nos últimos 30 anos, a farmácia privativa e as drogarias decifraram receitas (aqui está feita referência às letras de difícil leitura) eentregaram aos usuários do serviço os produtos correspondentes, quase sempre sem qualquer tipo de orientação sobre o uso dos mesmos. A manipulação de fórmulas também não fugiu a esta regra.

Em relação aos serviços públicos, os governos e dirigentes discutem a questão do abastecimento de medicamentos e as estratégias de financiamento, mas poucos reconhecem que os medicamentos são apenas um instrumento da prestação de um serviço e geralmente não se preocupam com a estruturação e a organização deste serviço ${ }^{26}$. A maioria das farmácias de unidades básicas de saúde funciona porque um leigo ou profissional com parcos conhecimentos sobre medicamentos atua na dispensação (auxiliares de enfermagem, auxiliares administrativos, de cozinha, entre outros).

Em meio a inúmeras necessidades e demandas, os serviços de farmácia não são considerados prioritários na disputa por recursos nos orçamentos da saúde. Talvez a sua importância ainda não esteja explicitada para a maioria dos gestores. Isto é possível constatar pelas condições físicas e de recursos humanos em que se encon- 
tram, embora estudos sobre o tema sejam ne cessários. Dentro da estrutura das unidades de saúde, a farmácia geralmente ocupa pequenos espaços, muitas vezes sem as condições mínimas necessárias para o armazenamento adequado de medicamentos. Ainda é possível encontrar farmácias em que há grades separando o usuário do serviço e o profissional que faz 0 atendimento. Além disso, falta pessoal qualificado. Assim, não há condições apropriadas para que este serviço desempenhe a sua função e para que de fato as relações sejam mais humanizadas.

Vale elembrar que a ausência deserviço defarmácia adequado, que zele pelo uso racional de medicamentos em parceria com os demais serviços e profissionais do sistema de saúde, constitui um problema importantedesaúde públicae, para chegarmos a esta conclusão, lembremos apenas das informações que tratam da morbimortalidade relacionada a medicamentos nos EUA, país em que todas as farmácias possuem farmacêuticos, e façamos um paralelo com a situação descrita anteriormente.

Faz-se necessário atentar para o uso racional dos medicamentos, de forma que os pacientes recebam osmedicamentos para a indicação apropriada, nas doses, via de administração e duração apropriadas; que não existam contra-indicações; que a probabilidade de ocorrência de re ações adversas seja mínima; que a dispensação seja correta eque haja aderência ao tratamento ${ }^{2}$.

Os serviços farmacêuticos de atenção primária contribuem para a diminuição da internação ou do tempo de permanência no hospital, à assistência aos portadores de doenças crônicas, à prática de educação em saúdee, para uma intervenção terapêutica mais custo- efetiva ${ }^{27}$.

Dentro desta lógica, o serviço defarmácia deve assumir papel complementar ao serviço médico na atenção à saúde. 0 paciente que sai do consultório com uma receita terá maior resolução de seus problemas se tiver acesso ao tratamento prescrito e se a prescrição atender à racionalidade terapêutica. M as não é só isto. Também é ne cessário avaliar os fatores que potencialmente podem interferir em seu tratamento, como: hábitos alimentares, tabagismo, histórico de reações alérgicas, uso de outros medicamentos ou drogas, outras doenças, etc. ou atémesmo a falta de adesão. Esta avaliação, com a possi bilidade de intervenção visando à efetividade terapêutica, pode ser alcançada com a implantação da atenção farmacêutica.

O farmacêutico, via de regra, éo último profissional de saúde que tem contato direto com o paciente depois da decisão médica pela terapia farmacológica ${ }^{28}$. Desta forma, torna-se co-responsável pela sua qualidade de vida. Tanto o usuário quanto o profissional devem ser vistos na totalidade do seu ser e por isso os conceitos de pessoa, responsabilidade, respeito, verdade, consciência, autonomia, justiça, etc. devem ser interiorizados para modelar a conduta profissional 29.

A humanização do serviço de farmácia passa por todos estes aspectos e também abrangequestões relativas ao ambiente de aten dimento. É necessário que haja instalações adequadas o suficiente para causar bem-estar e confiança. Que o farmacêutico possa atendê-lo em sala reservada para este fim, garantindo privacidade.

Assim, a estruturação das ações de atenção farmacêutica dentro do serviço defarmácia constitui uma abordagem imprescindível para a promoção da saúde. 0 quadro 1 relaciona algumas destas ações que podem ser adotadas para que este serviço seja reestruturado.

\section{Quadro 1}

A ções para a reorientação dos serviços de farmácia.

- Implantação de ações de atenção farmacêutica para:

- Aumentar a aderência ao tratamento;

- Prevenir intoxicações;

- Promover o uso e o armazenamento de forma segura;

- Prevenir o surgimento de problemas relacionados aos medicamentos;

- Disposição de instalações, ambientes e equipamentos adequados;

- Estabelecimento de manuais de boas práticas de armazenamento e dispensação;

- Capacitação dos funcionários da farmácia;

- Fornecimento de educação continuada aos demais profissionais de saúde para assuntos relativos a medicamentos;

- Redução de filas para 0 atendimento (principalmente no serviço público); - Oferta de informação com qualidade;

- Integração entre farmacêutico e equipe e, da farmácia aos demais serviços de saúde;

- Elaboração de educação em saúde e campanhas vinculadas às necessidades da comunidade (perfil epidemiológico);

- M elhora da qualidade da comunicação com o paciente. 
Desenvolvimento das habilidades da comunidade

O farmacêutico pode trabalhar para que a comunidade esteja informada sobre condições que sejam determinantes sobre o seu estado de saúde. A conscientização da comunidade é um pré-requisito para que sejam alcançados níveis elevados de saúde. É necessário que a mesma esteja orientada sobre como proceder em relação ao uso de medicamentos e conheça as doenças mais prevalentes em seu meio, bem como as maneiras para preveni-las ou minimizar suas complicações.

Com o desenvolvimento das habilidades individuais e da comunidade, será possível contar com seu apoio para a realização de movimentos maiores, que tenham em vista a promoção da saúde. 0 quadro 2 descreve algumas ações que podem ser adotadas para alcançar este objetivo.

Incentivo à ação comunitária

Incentivar a ação comunitária reforça todas as medidas adotadas para a promoção da saúde.
A comunidade passa a ser um forte aliado com vista à utilização racional demedicamentos, identificando os problemas mais freqüentes e compartilhando com o farmacêutico a responsabilidade pela divulgação da informação para todos os indivíduos.

\section{Consideraçõesfinais}

A profissão farmacêutica, do século XX até os dias de hoje, passou por vários momentos difíceis, inclusive experimentando a perda da identidade. Com a prática da atenção farmacêutica ea carência da população de um farmacêutico mais atuante em defesa do uso racional dos medicamentos, surge uma oportunidade ímpar para o desempenho de seu papel perante a sociedade.

Faz parte de suas atribuições a promoção da saúde, principalmente através da disposição de um serviço de farmácia com qualidade (e neste aspecto incluem-se a orientação eo acompanhamento farmacêutico) e, da educação em saúde, de fácil acesso à população.

0 uso irracional de medicamentos é um importante problema de saúde pública; portanto, é
Quadro 2

Ações para desenvolver as habilidades da comunidade.

- Identificação das necessidades da população/ comunidade em relação à informação em saúde (considerando as condições de alimentação, habitação, escolaridade, morbidade e mortalidade, higiene, etc. em que vivem os indivíduos);

- Elaboração de impressos abordando temas relativos ao acondicionamento, prazos de validade, efeitos e uso de medicamentos, bem como a importância da adesão ao tratamento; - Palestras sobre as doenças e os medicamentos, dirigidas a grupos específicos, como por exemplo, diabéticos, hipertensos, alcoólatras, pacientes HIV positivos, etc;

- Elaboração de campanhas para o

desenvolvimento do conceito de que o restabelecimento da saúde não se restringe à prática terapêutica medicamentosa, mas constitui conjunto de variáveis que devem ser observadas, como por exemplo, hábitos alimentares, de higiene, abuso de drogas lícitas ou ilícitas, etc.
Quadro 3

M edidas para incentivar à ação comunitária.

- Estabelecimento de prioridades de temas para a educação em saúde juntamente com a comunidade;

- Participação ativa de membros da comunidade em palestras que tratem sobre o medicamento, como exemplo, uso racional de medicamentos ou automedicação (utilizando espaços que estejam próximos à comunidade, como, sociedades amigos do bairro, igrejas, associações, sindicatos, etc.);

- Estímulo a que a comunidade participe de campanhas de saúde, como as de combate à dengue, vacinação de animais contra raiva, vacinação de crianças e adultos, orientação a que gestantes façam o pré-natal, etc., bem como atue apoiando e informando os membros da comunidade sobre os perigos das drogas de abuso, como os cigarros, álcool, cocaína, maconha, anfetaminas, entre outras;

- Estímulo à discussão sobre hábitos saudáveis de vida: alimentação, exercícios físicos, etc;

- Participação conjunta para desenvolver ações de prevenção das doenças. 
preciso considerar o potencial de contribuição do farmacêutico e efetivamente incorporá-lo às equipes de saúde a fim de que se garanta a meIhoria da utilização dos medicamentos, com redução dos riscos de morbimortal idade e que seu trabalho proporcione meios para que os custos relacionados à farmacoterapia sejam os menores possíveis para a sociedade.

\section{Referências}

1. Organização Pan-Americana de Saúde (OPS). Pautas para establecer políticas farmacéuticas nacionales. Ginebra: OM S; 1988.

2. Sociedade Brasileira de Vigilância de M edicamentos (Sobravime). 0 que é uso racional de medicamentos. São Paulo: Sobravime; 2001. p.50-56.

3. Flower R. Lifestyle drugs: pharmacology and the social agenda. Trends in Pharmacological Sciences 2004; 25: 182-5.

4. Abrafarma. Números Abrafarma. Perfil e comparativo de vendas nominais $2003 \times 2002$. [documento da Internet]. [acessado 2004 Ago 08]. Disponível em: <http://www.abrafarma.com.br/ numerosabrafarma.htm>

5. Ministério da Saúde. Execução Orçamentária e Financeira 2003. [documento da Internet]. [acessado 2003 Ago 06]. Disponível em: <http://www. saude.gov.br

6. Van de Bemt PM, Egberts TC, de Jong-Van den Berg LT, Brouwers JR 2000. Drug-related problems in hospitalized patients. Drug Saf 2000; 22(4): 321-33.

7. White TJ, Arakelian A, Rho JP. Counting the costs of the drug-related adverse events. PharmacoEconomics 1999; 15(5): 445-58.

8. Holland EG, Degruy FV. Drug-induced disorders. Am Fam Physician 1997;56(7): 1781-8.

9. Patel P, Zed PJ. Drug-related visits to the emergency department: how big is the problem? Pharmacotherapy 2002; 22(7): 915-23.

10. Sweeney MA. Physician-pharmacist collaboration: a millennial paradigm to reduce medication errors. Journal of American Osteopathology Association 2002;102(12): 678-81.
11. Hepler CD, Strand LM. Oportunidades y responsabilidades en la Atención Farmacéutica. Pharmaceutical Care España 1999; 1, p.35-47.

12. Lei Federal no 5.991, de 17 de dezembro de 1973. Dispõe sobre o controle sanitário do comércio de drogas, medicamentos, insumos farmacêuticos e correlatos, e dá outras providências. In: Conselho Regional de Farmácia do Estado de São Paulo. Legislação para o farmacêutico. São Paulo: CRF-SP; 1996. p.22-33.

13. Zubioli A . Profissão: farmacêutico. E agora? Curitiba: Editora Lovise;1992.

14. Barros JAC. Propaganda de medicamentos: atentado à saúde? São Paulo: Hucitec /Sobravime; 1995.

15. Lexchin J. U ma fraude planejada: a publicidade farmacêutica no terceiro mundo. In: Bonfim JRA, M ercucci VL. A construção da política de medicamentos. São Paulo: Hucitec/Sobravime; 1997. p.269-289.

16. Zubioli $A$, organizadores. A farmácia clínica na farmácia comunitária. Brasília: Ethosfarma; 2001.

17. Secretaria de Estado de Saúde de São Paulo (SESSP). Centro de Vigilância Sanitária. Programa de Farmacovigilância. Farmacovigilância: ação na reação. São Paulo: SES; 2002.

18. Organização Pan-Americana de Saúde (OPS). Promoción del uso racional de medicamentos: componentes centrales. Ginebra: OPS; 2002.

19. Laing R, et al. 25 years of the WHO essential medicines lists: progress and challenges. Lancet 2003; 361:1723-29.

20. Organização Mundial de Saúde (OMS). El papel del farmaceutico en el sistema de atención de salud. Tókio: OM S; 1993. 
21. Otero MJ, Dominguez-Gil A. Acontecimientos adversos por medicamentos: uma patologia emergente. Farm H osp 2000; 24(4): 258-266.

22. Organização Pan-Americana de Saúde (OPS). Proposta: consenso brasileiro de atenção farmacêutica. Atenção farmacêutica no Brasil: "trilhando caminhos". Brasília: OPAS; 2002.

23. James JA, Rovers JP. Wellness and health promotion. In: Rovers JP, et al. A practical guide to pharmaceutical care. Washington: American Pharmaceutical Association; 2003. p.183-200.

24. Organização Mundial de Saúde (OMS). Carta de Ottawa. Primeira Conferência Internacional sobre Promoção da Saúde. Ottawa; 1986. [documento da Internet]. [acessado 2003 Ago 03]. Disponível em: <http://www.opas.org.br/promoção/uploadArq/ Ottawa.pdf

25. Oshiro M L, Castro LLC. Avaliação dos efeitos de uma intervenção educativa para promoção do uso da terapia de reidratação oral (TRO) em trabalhadores de farmácias. Cad Saúde Pública 2002; 18(1) 287-297.
26. Marín NJ. Los servicios farmacéuticos en la atención de la salud. Boletín Farmacéutico Bonaerense 2002. [periódico na Internet]. [acessado 2004 Abr 05]. (350). Disponível em: <http://www.colfarma.org.ar/ boletin/revistas/articulos/n350/articulo350f.html

27. Marín $\mathrm{N}$, et al., organizadores. Assistência farmacêutica para gerentes municipais de saúde. Rio de Janeiro: OPAS/OM S; 2003. p. 239-286.

28. Ferraes AMB, Cordoni Jr L. M edicamento, farmácia, farmacêutico e o usuário: novo século, novas demandas; 2003. [documento da Internet]. [acessado 2003 Ago 03]. Disponível em: <http://www.ccs.uol.br/ espacoparasaude/v4n1/doc/farmacia.doc

29. M artins, M CFN. Humanização da assistência e formação profissional. In: Secretaria Municipal da Saúde de São Paulo. Área Temática de Assistência Farmacêutica. Termos de Referência da I Conferência M unicipal de Política de M edicamentos e Assistência Farmacêutica. São Paulo; 2002.

Artigo apresentado em 8/04/2005

Aprovado em 12/10/2005

Versão final apresentada em 29/11/2005 